Research Article

\title{
Free Vibration of Annular Circular Plates Based on Higher-Order Shear Deformation Theory: A Spline Approximation Technique
}

\author{
Saira Javed (iD) and F. H. H. Al Mukahal \\ Department of Mathematics and Statistics, College of Science, King Faisal University, P.O. Box 400, Hofuf 31982, Saudi Arabia \\ Correspondence should be addressed to Saira Javed; sulhaque@kfu.edu.sa
}

Received 17 June 2021; Revised 12 August 2021; Accepted 24 September 2021; Published 16 December 2021

Academic Editor: Rosario Pecora

Copyright (C) 2021 Saira Javed and F. H. H. Al Mukahal. This is an open access article distributed under the Creative Commons Attribution License, which permits unrestricted use, distribution, and reproduction in any medium, provided the original work is properly cited.

\begin{abstract}
This research is based on higher-order shear deformation theory to analyse the free vibration of composite annular circular plates using the spline approximation technique. Equilibrium equations are derived, and differential equations in terms of displacement and rotational functions are obtained. Cubic or quantic spline is used to approximate the displacement and rotational functions depending upon the order of these functions. A generalized eigenvalue problem is obtained and solved numerically for eigenfrequency parameter and associated eigenvector of spline coefficients. Frequency of annular circular plates with different numbers of layers with each layer consisting of different materials is analysed. The effect of geometric and material parameters on frequency value is investigated for simply supported condition. A comparative study with existing results narrates the validity of the present results. Graphs and tables depict the obtained results. Some figures and graphs are drawn by using Autodesk Maya and Matlab software.
\end{abstract}

\section{Introduction}

There are different kinds of plates like rectangular, circular, and annular circular have been used as structural elements of numerous engineering fields such as nuclear, civil, mechanical, aerospace, and marine. Any plate consisting of different layers forms composite. These layers can be of the same or different materials. The composite laminates are the source of attraction for engineers to design economical lightweight structures.

Different researchers have proposed different theories depending on the inclusion of shear deformation and rotary inertia in their formulations. Love-Kirchhoff proposed the classical theory which was based on the assumption that the straight lines normal to the undeformed and deformed midplane remain straight and normal and do not undergo stretching in the thickness direction. This theory accurately measures the stress analysis of thin composites plates but suitable for thick laminated plates since it overpredicts the natural frequencies $[1,2]$. Moreover, the study of relatively thick plates should account shear deformation. To fill this gap, Yang et al. [3] proposed the first order shear deformation theory (FSDT); according to them, there is a state of constant shear strain through the thickness of the plate (transverse shear strain). However, according to 3D elasticity theory, the shear strains vary at least quadratically through the thickness (transverse shear strain). Therefore, shear correction factors were introduced to correct the discrepancy in the shear forces of FSDT and 3D elasticity theory $[4,5]$. Moreover, a number of higher-order plate theories were developed to accurately evaluate the transverse shear stresses which effectively exists in the thick plates. In higher-order plate theories, the displacements are expanded up to any desired degree in terms of thickness coordinates $[1,2]$. In third-order plate theory, the displacement is expanded up to the cubic term in thickness coordinates to have quadratic variation of transverse shear strains and transverse shear stresses through the plate thickness. This avoids the need for shear correction coefficient [6]. The third-order plate TSDT theory of Reddy is widely used because it can represent transverse shear stresses in an efficient way. The inclusion of TSDT to equilibrium equations makes the solution more complex. These theories the classical plate theory (CPT), first-order shear deformation theory (FSDT), and higher-order shear deformation theories (HSDT) are shown 
in Figure 1. Novelty of the present work is based upon above discussion that the purpose of using HSDT to analyse composite plates is that it can accurately calculate shear stresses through the plate thickness.

Free vibration of annular circular plates is investigated by the number of researchers mostly using CPT and different numerical methods. Leissa [7-10] did a significant research on free vibration of annular circular plates. Dongxing and Yanhui [11] studied free vibration of beams. Azaripour and Baghani [12] examined the vibration of annular sector plates. Moreover, Xue et al. [13] studied free vibration of FG plates. Zhong et al. [14] also investigated the vibration of composites circular, annular, and sector plates, whereas Arshid and Khorshidvand [15] analyse the free vibration analysis of saturated porous FG circular plates using a differential quadrature method. Żur [16] examined free vibration analysis of elastically supported functionally graded annular plates using quasi-Green's function method. Kim and Dickinson [17] examined the vibration of annular circular plates under complicating effects. Different numerical methods are used to analyse annular circular plates: Kim and Dickinson [18] used the Rayleigh-Ritz method, Wang et al. [19] used the differential quadrature method, Liu and Chen [20] used the finite element method, and Gupta et al. [21] used the Chebyshev collocation technique. However, different boundary conditions were used to study the free vibration of annular circular plates [22-24]. Furthermore, isotropic annular circular plates were studied using the exact element method by Efraim and Eisenberger [25], and an annular circular plate attached to a free rigid core was studied by Wang [26]. Moreover, annular circular plates resting on elastic foundation were studied by Celep and Turhan [27], Utku et al. [28], and Kukla and Szewczyk [29]; Singh and Jain $[30,31]$ examined the free vibration annular circular plate on elastic edges. Free vibration of annular circular plates resting on Pasternak foundation was studied by Tajeddini et al. [32]. The discrete singular convolution method and differential quadrature method were used to examine free vibration of curved structural components such as truncated conical, cylindrical shells, and annular plates based on higher-order shear deformation theory [33]. Tornabene [34] and Tornabene et al. [35] investigated FG conical plates, cylindrical shells, and annular plates using a generalized differential quadrature method. Moving Kring interpolation was used to analyse vibration of plates based on HSDT by Vu et al. [36]. Composite plates were analysed by Thai et al. [37] using the finite element method and HSDT, whereas FSDT was used by Hosseini-Hashemi et al. [38] to analyse free vibration of rectangular plates. Frequency equations of annular circular plates were studied by Hashemi et al. [39]. Nguyen et al. [40] examined the vibration of functionally graded plates using HSDT. Moreover, nanoplates in the thermal environment were studied by Daikh et al. [41]. Baghlani et al. [42] investigated the free vibration of FGM cylindrical shells on elastic foundation. The FEM method was used to study the free and static vibration of FGM plates by Katili et al. [43]. Cross-ply laminates were studied by Dhari [44]. Higher-order shear deformation theories with a unified model were studied for composite plates by Li et al.

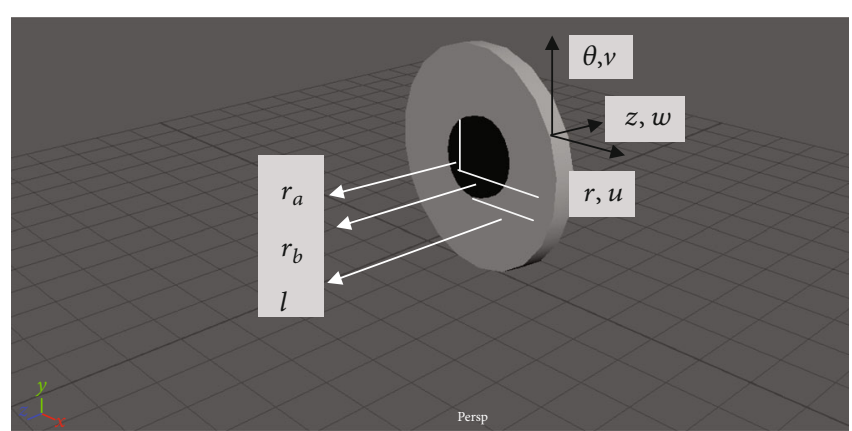

FIgURE 1: Various deformation theories.

[45]. Askari et al. [46] analysed the free vibration of eccentric annular plates. Amabili et al. [47] investigated annular plates coupled with fluids.

The present study can be a benchmark for researchers to verify future results of free vibration of annular circular plates. The spline method was used by Javed et al. [48-51] to solve the free vibrational problems of plates based on the first-order shear deformation theory and higher-order shear deformation theory. The aim of present study is to find the solution of engineering problem using TSDT which makes the calculation more complex but precise. Therefore, the purpose of this research is to examine the free vibration of cross-ply laminated annular circular plates using spline approximation technique. The kinematics of annular circular plates is based on TSDT. Displacement and rotational functions are approximated by cubic and quintic splines. Collocation with these splines yields a set of field equations which, along with the equations of boundary conditions, reduce to system of homogeneous simultaneous algebraic equations on the assumed spline coefficients. Then, the problem is solved using an eigensolution technique to obtain the frequency parameter. The eigenvectors are the spline coefficients from which the mode shapes are constructed. The frequency of annular circular plates was studied by circumferential node number, radius ratio, stacking sequence, and different lamination materials for simply supported boundary condition. Graphs and tables narrate the obtained results. Some figures and graphs are drawn by using Autodesk Maya and Matlab software's.

Usefulness of current research can be viewed from the fact that composite materials are usually used if the application needs high strength but also low weight. Most of engineering components are made up of composites because of their lightweight, strength, and possible association of material. The natural frequencies of any component must be known to avoid the destructive effect of weather and resonance with adjacent rotating or oscillating equipment. High specific strength and high specific stiffness are attractive features for the aerospace, and other automotive industries could be achieved by proper choice of composite materials. Composite structures offer better strength, temperature resistance, high damping, and resistance to corrosion, and the required properties could be achieved through variation of lamina orientation and stacking sequences, which provides an additional degree of flexibility to the designer, resulting in structures with tremendous potential. 


\section{Formulation}

Figure 2 shows the geometry of the annular circular plate. The curvilinear coordinate system $(r, \theta, z)$ is fixed at its reference surface, which is taken to be its middle surface. Here, $r_{a}=a$ is the inner radius and $r_{b}=b$ is the outer radius of the annular circular plate and $l=b-a$ is the width of the annular circular plate.

The displacement field considered is based on the thirdorder shear deformation theory [6] and is obtained by subtracting function $4 z^{3} / 3 h^{2}\left(\phi_{r}+\left(\partial w_{0} / \partial r\right)\right)$ and $4 z^{3} / 3 h^{2}$ $\left(\phi_{\theta}+\left(\partial w_{0} / \partial \theta\right)\right)$ from $u, v$ of Toorani and Lakis [52].

The stress resultants are defined as

$$
\begin{aligned}
\left\{\begin{array}{c}
N_{r} \\
N_{\theta} \\
N_{r \theta}
\end{array}\right\} & =\int_{-h / 2}^{h / 2}\left\{\begin{array}{c}
\sigma_{r} \\
\sigma_{\theta} \\
\sigma_{r \theta}
\end{array}\right\} d z,\left\{\begin{array}{c}
M_{r} \\
M_{\theta} \\
M_{r \theta}
\end{array}\right\} \\
& =\int_{-h / 2}^{h / 2} z\left\{\begin{array}{c}
\sigma_{r} \\
\sigma_{\theta} \\
\sigma_{r \theta}
\end{array}\right\} d z,\left\{\begin{array}{c}
P_{r} \\
P_{\theta} \\
P_{r \theta}
\end{array}\right\} \\
& =\int_{-h / 2}^{h / 2} z^{3}\left\{\begin{array}{c}
\sigma_{r} \\
\sigma_{\theta}
\end{array}\right\} d z,\left\{\begin{array}{l}
Q_{\theta} \\
Q_{r}
\end{array}\right\} \\
& =\int_{-h / 2}^{h / 2}\left\{\begin{array}{c}
\tau_{\theta z} \\
\tau_{r z}
\end{array}\right\} d z,\left\{\begin{array}{l}
R_{\theta} \\
R_{r}
\end{array}\right\} \\
& =\int_{-h / 2}^{h / 2} z^{2}\left\{\begin{array}{c}
\tau_{\theta z} \\
\tau_{r z}
\end{array}\right\} d z,
\end{aligned}
$$

where $N, M$, and $Q$ are stress, moment, and shear resultants, respectively, and $P$ and $R$ denote higher-order stress resultants.

The stress-strain relations are obtained as follows:

$$
\begin{gathered}
\left\{\begin{array}{c}
N_{r} \\
N_{\theta} \\
N_{r \theta} \\
M_{r} \\
M_{\theta} \\
M_{r \theta} \\
P_{r} \\
P_{\theta} \\
P_{r \theta}
\end{array}\right\}=\left(\begin{array}{lllllllll}
A_{11} & A_{12} & A_{16} & B_{11} & B_{12} & B_{16} & E_{11} & E_{12} & E_{16} \\
A_{12} & A_{22} & A_{26} & B_{12} & B_{22} & B_{26} & E_{12} & E_{22} & E_{26} \\
A_{16} & A_{26} & A_{66} & B_{16} & B_{26} & B_{66} & E_{16} & E_{26} & E_{66} \\
B_{11} & B_{12} & B_{16} & D_{11} & D_{12} & D_{16} & F_{11} & F_{12} & F_{16} \\
B_{12} & B_{22} & B_{26} & D_{12} & D_{22} & D_{26} & F_{12} & F_{22} & F_{26} \\
B_{16} & B_{26} & B_{66} & D_{16} & D_{26} & D_{66} & F_{16} & F_{26} & F_{66} \\
E_{11} & E_{12} & E_{16} & F_{11} & F_{12} & F_{16} & H_{11} & H_{12} & H_{16} \\
E_{12} & E_{22} & E_{26} & F_{12} & F_{22} & F_{26} & H_{12} & H_{22} & H_{26} \\
E_{16} & E_{26} & E_{66} & F_{16} & F_{26} & F_{66} & H_{16} & H_{26} & H_{66}
\end{array}\right)\left\{\begin{array}{c}
(0) \\
\varepsilon_{r} \\
(0) \\
\varepsilon_{\theta} \\
(0) \\
\gamma_{r \theta} \\
(1) \\
\varepsilon_{r} \\
(1) \\
\varepsilon_{\theta} \\
(1) \\
\gamma_{r \theta} \\
(3) \\
\varepsilon_{r} \\
(3) \\
\varepsilon_{\theta} \\
(3) \\
\gamma_{r \theta}
\end{array}\right\}, \\
\left\{\begin{array}{l}
Q_{\theta} \\
Q_{r} \\
R_{\theta} \\
R_{r}
\end{array}\right\}=\left\{\begin{array}{lllll}
A_{44} & A_{45} & D_{44} & D_{45} \\
A_{45} & A_{55} & D_{45} & D_{55} \\
D_{44} & D_{45} & F_{44} & F_{45} \\
D_{45} & D_{55} & F_{45} & F_{55}
\end{array}\right)\left\{\begin{array}{c}
(0) \\
\gamma_{\theta z} \\
\gamma_{r z} \\
\gamma_{\theta z} \\
\gamma_{\theta z} \\
(2) \\
\gamma_{r z}
\end{array}\right\},
\end{gathered}
$$

where $\varepsilon$ are strains and $\gamma$ are the shear strain components.

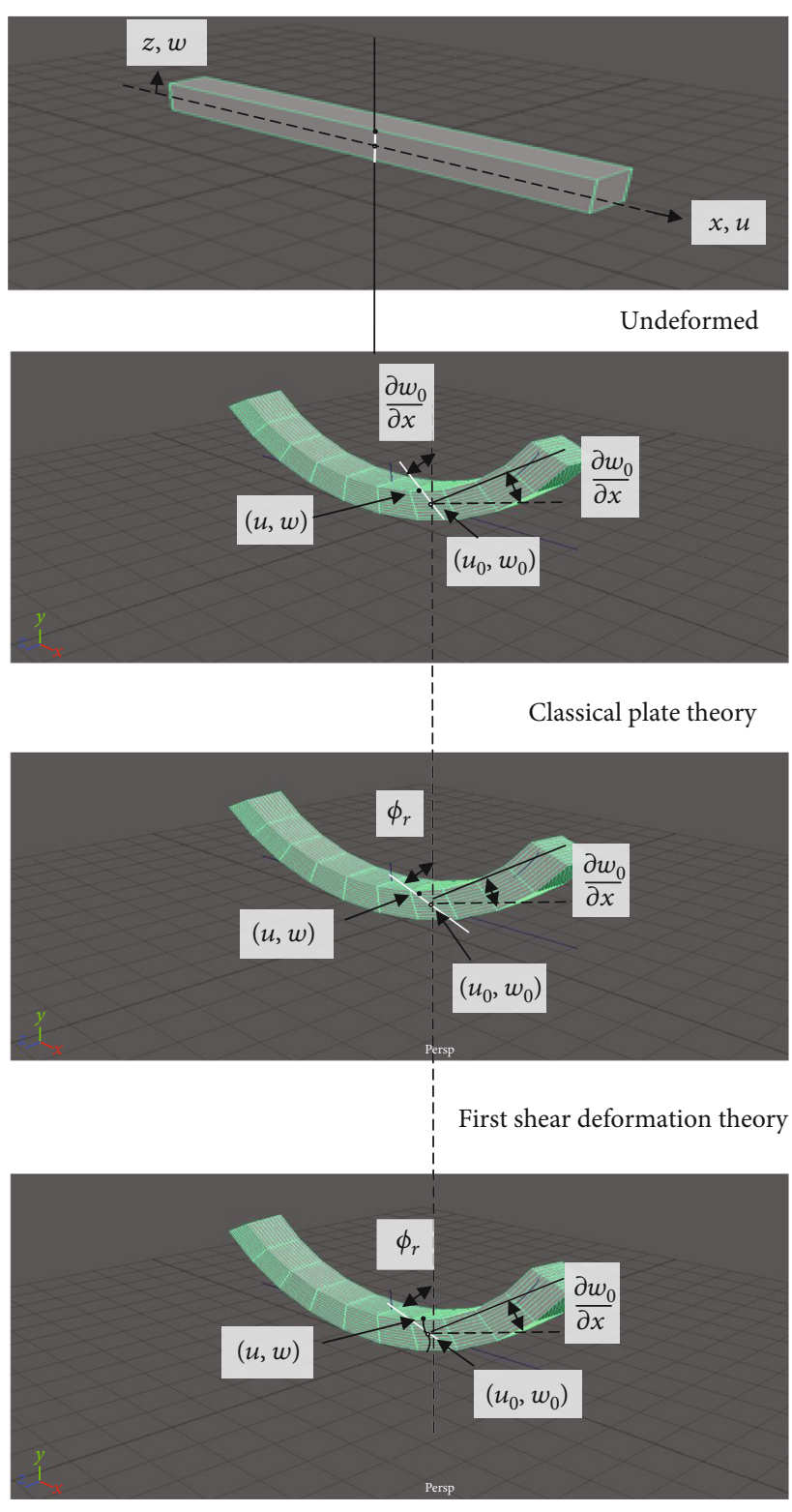

Higher shear deformation theory

Figure 2: The geometry of annular circular plate.

Stiffness coefficients $A_{i j}, B_{i j}$, and $D_{i j}$ (extensional, bending-extensional coupling, and bending stiffnesses) and higher-order stiffness coefficients $E_{i j}, F_{i j}$, and $H_{i j}$ are defined in Appendix A.

The equilibrium equations for annular circular plates considered are as follows:

$$
\begin{gathered}
\frac{\partial N_{r}}{\partial r}+\frac{1}{r}\left(N_{r}-N_{\theta}\right)+\frac{1}{r} \frac{\partial N_{r \theta}}{\partial \theta}=I_{0} \ddot{u}+J_{1} \ddot{\phi}_{r}-c_{1} I_{3} \frac{\partial \ddot{w}}{\partial r}, \\
\frac{\partial N_{r \theta}}{\partial r}+\frac{2}{r} N_{r \theta}+\frac{1}{r} \frac{\partial N_{\theta}}{\partial \theta}=I_{0} \ddot{v}+J_{1} \ddot{\phi}_{\theta}-c_{1} I_{3} \frac{\partial \ddot{w}}{\partial \theta},
\end{gathered}
$$




$$
\begin{gathered}
\frac{\partial \bar{Q}_{r}}{\partial r}+\frac{1}{r} \bar{Q}_{r}+\frac{1}{r} \frac{\partial \bar{Q}_{\theta}}{\partial \theta}+c_{2}\left(\frac{\partial^{2} P_{r}}{\partial r^{2}}+2 \frac{1}{r} \frac{\partial^{2} P_{r \theta}}{\partial r \partial \theta}+\frac{1}{r^{2}} \frac{\partial^{2} P_{\theta}}{\partial \theta^{2}}\right) \\
=c_{1} I_{3} \frac{\partial \ddot{u}}{\partial r}+\left(c_{1} I_{3}\right) \frac{\partial \ddot{v}}{\partial \theta}+I_{0} \ddot{w}-c_{1}^{2} I_{6}\left(\frac{\partial^{2} \ddot{w}}{\partial r^{2}}+\frac{\partial^{2} \ddot{w}}{\partial \theta^{2}}\right) \\
+c_{1} J_{4} \frac{\partial \ddot{\phi}_{r}}{\partial r}+c_{1} J_{4} \frac{\partial \ddot{\phi}_{\theta}}{\partial \theta}, \\
\frac{\partial \bar{M}_{r}}{\partial r}+\frac{1}{r}\left(\bar{M}_{r}-\bar{M}_{\theta}\right)+\frac{1}{r} \frac{\partial \bar{M}_{r \theta}}{\partial \theta}-\bar{Q}_{r}=J_{1} \ddot{u}-c_{1} J_{4} \frac{\partial \ddot{w}}{\partial r}+K_{2} \ddot{\phi}_{r}, \\
\frac{\partial \bar{M}_{r \theta}}{\partial r}+\frac{2}{r} \bar{M}_{r \theta}+\frac{1}{r} \frac{\partial \bar{M}_{\theta}}{\partial \theta}-\bar{Q}_{\theta}=J_{1} \ddot{v}-c_{1} J_{4} \frac{\partial \ddot{w}}{\partial \theta}+K_{2} \ddot{\phi}_{\theta}, \quad(3)
\end{gathered}
$$

where

$$
I_{i}=\int_{z} \rho^{(k)}(z)^{i} d z(i=0,1,2,3, \cdots, 6)
$$

where $\rho$ is the material density of the $k$-th layer, and

$$
\begin{gathered}
\bar{M}_{r}=M_{r}-c_{1} P_{r}, \bar{M}_{\theta}=M_{\theta}-c_{1} P_{\theta}, \bar{M}_{r \theta}=M_{r \theta}-c_{1} P_{r \theta}, \\
\bar{Q}_{\theta}=Q_{\theta}-c_{2} R_{\theta}, \bar{Q}_{r}=Q_{r}-c_{2} R_{r}, \\
J_{1}=I_{1}-c_{1} I_{3}, \\
J_{4}=I_{4}-c_{1} I_{6}, \\
K_{2}=I_{2}-2 c_{1} I_{4}+c_{1}^{2} I_{6},
\end{gathered}
$$

where

$$
\begin{gathered}
c_{1}=\frac{4}{h^{2}}, c_{2}=\frac{c_{1}}{3} \\
\left(A_{11} \frac{\partial^{2}}{\partial r^{2}}+A_{11} \frac{1}{r} \frac{\partial}{\partial r}-A_{22} \frac{1}{r^{2}}+A_{66} \frac{1}{r^{2}} \frac{\partial^{2}}{\partial \theta^{2}}\right) U \\
+\left(\left(A_{12}+A_{66}\right) \frac{1}{r} \frac{\partial^{2}}{\partial r \partial \theta}-\left(A_{22}+A_{66}\right) \frac{1}{r^{2}} \frac{\partial}{\partial \theta}\right) V \\
-\left(E_{11} c_{2} \frac{\partial^{3}}{\partial r^{3}}+\left(E_{11} c_{2}-E_{12} c_{2}\right) \frac{1}{r} \frac{\partial^{2}}{\partial r^{2}}\right. \\
\left.+\left(E_{12} c_{2}-2 E_{66} c_{2}\right) \frac{1}{r^{2}} \frac{\partial^{3}}{\partial r \partial \theta^{2}}-\left(E_{12} c_{2}+E_{22} c_{2}\right) \frac{1}{r^{3}} \frac{\partial^{2}}{\partial \theta^{2}}\right) W \\
+\left(\left(B_{11}-E_{11} c_{2}\right) \frac{\partial^{2}}{\partial r^{2}}+\left(B_{11}-E_{11} c_{2}\right) \frac{1}{r} \frac{\partial}{\partial r}\right. \\
\left.+\left(B_{66}-E_{66} c_{2}\right) \frac{1}{r^{2}} \frac{\partial^{2}}{\partial \theta^{2}}-\left(B_{22}-E_{22} c_{2}\right) \frac{1}{r^{2}}\right) \phi_{r} \\
-\left(\left(B_{22}-E_{22} c_{2}+B_{66}-E_{66} c_{2}\right) \frac{1}{r^{2}} \frac{\partial}{\partial \theta}\right. \\
\left.-\left(B_{12}-E_{12} c_{2}+B_{66}-E_{66} c_{2}\right) \frac{1}{r} \frac{\partial^{2}}{\partial r \partial \theta}\right) \phi_{\theta} \\
=I_{0} \ddot{u}+J_{1} \ddot{\phi}_{r}-c_{1} I_{3} \frac{\partial \ddot{w}}{\partial r},
\end{gathered}
$$

$$
\begin{aligned}
\left(\left(A_{66}\right.\right. & \left.\left.+A_{22}\right) \frac{1}{r^{2}} \frac{\partial}{\partial \theta}+\left(A_{66}+A_{12}\right) \frac{1}{r} \frac{\partial^{2}}{\partial r \partial \theta}\right) U \\
& +\left(A_{66} \frac{\partial^{2}}{\partial r^{2}}+A_{66} \frac{1}{r} \frac{\partial}{\partial r}+A_{22} \frac{1}{r^{2}} \frac{\partial^{2}}{\partial \theta^{2}}-A_{66} \frac{1}{r^{2}}\right) V \\
& -\left(\left(2 E_{66} c_{2}+E_{12} c_{2}\right) \frac{1}{r} \frac{\partial^{3}}{\partial r^{2} \partial \theta}-2 E_{66} c_{2} \frac{1}{r^{2}} \frac{\partial^{2}}{\partial r \partial \theta}-E_{22} c_{2} \frac{1}{r^{3}} \frac{\partial^{3}}{\partial \theta^{3}}\right) W \\
& +\left(\left(B_{66}-E_{66} c_{2}+B_{12}-E_{12} c_{2}\right) \frac{1}{r} \frac{\partial^{2}}{\partial r \partial \theta}+\left(B_{66}-E_{66} c_{2}+B_{22}-E_{22} c_{2}\right) \frac{1}{r^{2}} \frac{\partial}{\partial \theta}\right) \phi_{r} \\
& +\left(\left(B_{66}-E_{66} c_{2}\right) \frac{\partial^{2}}{\partial r^{2}}+\left(B_{66}-E_{66} c_{2}\right) \frac{1}{r} \frac{\partial}{\partial r}\right. \\
& \left.+\left(B_{22}-E_{22} c_{2}\right) \frac{1}{r^{2}} \frac{\partial^{2}}{\partial \theta^{2}}-\left(B_{66}-E_{66} c_{2}\right) \frac{1}{r^{2}}\right) \phi_{\theta} \\
= & I_{0} \ddot{v}+J_{1} \ddot{\phi}_{\theta}-c_{1} I_{3} \frac{\partial \ddot{w}}{\partial \theta},
\end{aligned}
$$

$$
\begin{aligned}
\left(E_{11} c_{1}\right. & \frac{\partial^{3}}{\partial r^{3}}+E_{12} c_{1} \frac{1}{r} \frac{\partial^{2}}{\partial r^{2}}-2 E_{12} c_{1} \frac{1}{r^{2}} \frac{\partial}{\partial r} \\
& \left.+\left(E_{12} c_{1}+2 E_{66} c_{1}\right) \frac{1}{r^{2}} \frac{\partial^{3}}{\partial r \partial \theta^{2}}+\left(E_{22} c_{1}-2 E_{66} c_{1}\right) \frac{1}{r^{3}} \frac{\partial^{2}}{\partial \theta^{2}}-2 E_{12} c_{1} \frac{1}{r^{3}}\right) U \\
& +\left(\left(E_{12} c_{1}+2 E_{66} c_{1}\right) \frac{1}{r} \frac{\partial^{3}}{\partial r^{2} \partial \theta}-\left(2 E_{12} c_{1}+2 E_{66} c_{1}\right) \frac{1}{r^{2}} \frac{\partial^{2}}{\partial r \partial \theta}\right. \\
& \left.+E_{22} c_{1} \frac{1}{r^{3}} \frac{\partial^{3}}{\partial \theta^{3}}+2\left(E_{12} c_{1}+E_{66} c_{1}\right) \frac{1}{r^{3}} \frac{\partial}{\partial \theta}\right) V \\
& +\left(-H_{11} c_{1}^{2} \frac{\partial^{4}}{\partial r^{4}}+\left(A_{55}-2 D_{55} c_{2}+F_{55} c_{2}^{2}\right) \frac{\partial^{2}}{\partial r^{2}}\right. \\
& +\left(A_{55}-2 D_{55} c_{2}+F_{55} c_{2}^{2}\right) \frac{1}{r} \frac{\partial}{\partial r}-\left(2 H_{12} c_{1}^{2}+4 H_{66} c_{1}^{2}\right) \frac{1}{r^{2}} \frac{\partial^{4}}{\partial r^{2} \partial \theta^{2}} \\
& +\left(4 H_{12} c_{1}^{2}+4 H_{66} c_{1}^{2}\right) \frac{1}{r^{3}} \frac{\partial^{3}}{\partial r \partial \theta^{2}}-H_{22} c_{1}^{2} \frac{1}{r^{4}} \frac{\partial^{4}}{\partial \theta^{4}}
\end{aligned}
$$$$
\left.+\left(\left(A_{55}-2 D_{55} c_{2}+F_{55} c_{2}^{2}\right) \frac{1}{r^{2}}-6 H_{12} c_{1}^{2} \frac{1}{r^{4}}\right) \frac{\partial^{2}}{\partial \theta^{2}}\right) W
$$$$
+\left(\left(F_{11} c_{2}-H_{11} c_{1}^{2}\right) \frac{\partial^{3}}{\partial r^{3}}+\left(F_{12} c_{1}-H_{12} c_{1}^{2}\right) \frac{1}{r} \frac{\partial^{2}}{\partial r^{2}}\right.
$$$$
+\left(\left(A_{55}-2 D_{55} c_{2}+F_{55} c_{2}^{2}\right)-2\left(F_{12} c_{1}-H_{12} c_{1}^{2}\right) \frac{1}{r^{2}}\right) \frac{\partial}{\partial r}
$$$$
+\left(\left(F_{12} c_{1}-H_{12} c_{1}^{2}\right)+2\left(F_{66} c_{1}-H_{66} c_{1}^{2}\right)\right) \frac{1}{r^{2}} \frac{\partial^{3}}{\partial r \partial \theta^{2}}
$$$$
+\left(\left(F_{22} c_{1}-H_{22} c_{1}^{2}\right)-2\left(F_{66} c_{1}-H_{66} c_{1}^{2}\right)\right) \frac{1}{r^{3}} \frac{\partial^{2}}{\partial \theta^{2}}
$$$$
\left.+\left(A_{55}-2 D_{55} c_{2}+F_{55} c_{2}^{2}\right) \frac{1}{r}+2\left(F_{12} c_{1}-H_{12} c_{1}^{2}\right) \frac{1}{r^{3}}\right) \phi_{r}
$$$$
+\left(\left(\left(F_{12} c_{1}-H_{12} c_{1}^{2}\right)+2\left(F_{66} c_{1}-H_{66} c_{1}^{2}\right)\right) \frac{1}{r} \frac{\partial^{3}}{\partial r^{2} \partial \theta}\right)
$$$$
-\left(\left(2\left(F_{12} c_{1}-H_{12} c_{1}^{2}\right)+2\left(F_{66} c_{1}-H_{66} c_{1}^{2}\right)\right) \frac{1}{r^{2}}\right) \frac{\partial^{2}}{\partial r \partial \theta}
$$$$
\cdot\left(\left(A_{44}-2 D_{44} c_{2}+F_{44} c_{2}^{2}\right) \frac{1}{r}+\left(2\left(F_{12} c_{1}-H_{12} c_{1}^{2}\right)\right.\right.
$$$$
\left.\left.\left.+2\left(F_{66} c_{1}-H_{66} c_{1}^{2}\right)\right) \frac{1}{r^{3}}\right) \frac{\partial}{\partial \theta}+\left(F_{22} c_{1}-H_{22} c_{1}^{2}\right) \frac{1}{r^{3}} \frac{\partial^{3}}{\partial \theta^{3}}\right) \phi_{\theta}
$$$$
=c_{1} I_{3} \frac{\partial \ddot{u}}{\partial r}+\left(c_{1} I_{3} \frac{\partial \ddot{v}}{\partial \theta}+I_{0} \ddot{w}\right.
$$$$
-c_{1}^{2} I_{6}\left(\frac{\partial^{2} \ddot{w}}{\partial r^{2}}+\frac{\partial^{2} \ddot{w}}{\partial \theta^{2}}\right)+c_{1} J_{4} \frac{\partial \ddot{\phi}_{r}}{\partial r}+c_{1} J_{4} \frac{\partial \ddot{\phi}_{\theta}}{\partial \theta},
$$ 


$$
\begin{aligned}
\left(\left(B_{11}\right.\right. & \left.-E_{11} c_{1}\right) \frac{\partial^{2}}{\partial r^{2}}+\left(B_{11}-E_{11} c_{1}\right) \frac{1}{r} \frac{\partial}{\partial r} \\
& \left.+\left(B_{66}-E_{66} c_{1}\right) \frac{1}{r^{2}} \frac{\partial^{2}}{\partial \theta^{2}}-\left(B_{22}-E_{22} c_{1}\right) \frac{1}{r^{2}}\right) U \\
& +\left(\left(B_{12}-E_{12} c_{1}+B_{66}-E_{66} c_{1}\right) \frac{1}{r} \frac{\partial^{2}}{\partial r \partial \theta}\right. \\
& \left.-\left(B_{22}-E_{22} c_{1}+B_{66}-E_{66} c_{1}\right) \frac{1}{r^{2}} \frac{\partial}{\partial \theta}\right) V \\
& -\left(\left(F_{11} c_{1}-H_{11} c_{1}^{2}\right) \frac{\partial^{3}}{\partial r^{3}}+\left(F_{11} c_{1}-H_{11} c_{1}^{2}-F_{12} c_{1}+H_{12} c_{1}^{2}\right) \frac{1}{r} \frac{\partial^{2}}{\partial r^{2}}\right. \\
& +\left(A_{55}-2 D_{55} c_{2}+F_{55} c_{2}^{2}\right) \frac{\partial}{\partial r}+\left(F_{12} c_{1}-H_{12} c_{1}^{2}+2 F_{66} c_{1}-2 H_{66} c_{1}^{2}\right) \frac{1}{r^{2}} \frac{\partial^{3}}{\partial r \partial \theta^{2}} \\
& \left.-\left(F_{12} c_{1}-H_{12} c_{1}^{2}+F_{22} c_{1}-H_{22} c_{1}^{2}\right) \frac{1}{r^{3}} \frac{\partial^{2}}{\partial \theta^{2}}\right) W \\
& \cdot\left(\left(D_{11}-2 F_{11} c_{1}+H_{11} c_{1}^{2}\right) \frac{\partial^{2}}{\partial r^{2}}+\left(D_{11}-2 F_{11} c_{1}+H_{11} c_{1}^{2}\right) \frac{1}{r} \frac{\partial}{\partial r}\right. \\
& +\left(D_{66}-2 F_{66} c_{1}+H_{66} c_{1}^{2}\right) \frac{1}{r^{2}} \frac{\partial^{2}}{\partial \theta^{2}}-\left(D_{22}-2 F_{22} c_{1}\right. \\
& \left.\left.+J_{1} \ddot{u}-c_{1} J_{4} \frac{\partial \ddot{w}}{\partial r}+K_{2} \ddot{\phi}_{1}^{2}\right) \frac{1}{r^{2}}-\left(A_{55}-2 D_{55} c_{2}+F_{55} c_{2}^{2}\right)\right) \phi_{r} \\
& +\left(\left(D_{12}-2 F_{12} c_{1}+H_{12} c_{1}^{2}+D_{66}-2 F_{66} c_{1}+H_{66} c_{1}^{2}\right) \frac{1}{r} \frac{\partial^{2}}{\partial r \partial \theta}\right. \\
& \left.\left(D_{22}-2 F_{22} c_{1}+H_{22} c_{1}^{2}+D_{66}-2 F_{66} c_{1}+H_{66} c_{1}^{2}\right) \frac{1}{r^{2}} \frac{\partial}{\partial \theta}\right) \phi_{\theta}
\end{aligned}
$$

$$
\begin{aligned}
\left(\left(B_{66}\right.\right. & \left.\left.-E_{66} c_{1}+B_{12}-E_{12} c_{1}\right) \frac{1}{r} \frac{\partial^{2}}{\partial r \partial \theta}+\left(B_{66}-E_{66} c_{1}+B_{22}-E_{22} c_{1}\right) \frac{1}{r^{2}} \frac{\partial}{\partial \theta}\right) U \\
& +\left(\left(B_{66}-E_{66} c_{1}\right) \frac{\partial^{2}}{\partial r^{2}}+\left(B_{66}-E_{66} c_{1}\right) \frac{1}{r} \frac{\partial}{\partial r}\right. \\
& \left.+\left(\left(B_{22}-E_{22} c_{1}\right)\right) \frac{1}{r^{2}} \frac{\partial^{2}}{\partial \theta^{2}}-\left(\left(B_{66}-E_{66} c_{1}\right)\right) \frac{1}{r^{2}}\right) V \\
& -\left(\left(2 F_{66} c_{1}-2 H_{66} c_{1}^{2}+F_{12} c_{1}-H_{12} c_{1}^{2}\right) \frac{1}{r} \frac{\partial^{3}}{\partial r^{2} \partial \theta}\right. \\
& +2\left(F_{66} c_{1}-H_{66} c_{1}^{2}\right) \frac{1}{r^{2}} \frac{\partial^{2}}{\partial r \partial \theta}+\left(F_{22} c_{1}-H_{22} c_{1}^{2}\right) \frac{1}{r^{3}} \frac{\partial^{3}}{\partial \theta^{3}} \\
& \left.-\left(A_{44}-2 D_{44} c_{2}+F_{44} c_{2}^{2}\right) \frac{1}{r} \frac{\partial}{\partial \theta}\right) W \\
+ & \left(\left(D_{66}-2 F_{66} c_{1}+H_{66} c_{1}^{2}+D_{12}-2 F_{12} c_{1}+H_{12} c_{1}^{2}\right) \frac{1}{r} \frac{\partial^{2}}{\partial r \partial \theta}\right. \\
+ & \left.\left(D_{66}-2 F_{66} c_{1}+H_{66} c_{1}^{2}+D_{22}-2 F_{22} c_{1}+H_{22} c_{1}^{2}\right) \frac{1}{r^{2}} \frac{\partial}{\partial \theta}\right) \phi_{r} \\
+ & J_{1} \ddot{v}-c_{1} J_{4} \frac{\partial \ddot{w}}{\partial \theta}+K_{2} \ddot{\phi}_{\theta} . \\
+ & \left(\left(D_{66}-2 F_{66} c_{1}+H_{66} c_{1}^{2}\right) \frac{\partial^{2}}{\partial r^{2}}+\left(D_{66}-2 F_{66} c_{1}+H_{66} c_{1}^{2}\right) \frac{1}{r} \frac{\partial}{\partial r}\right. \\
+ & \left(D_{22}-2 F_{22} c_{1}+H_{22} c_{1}^{2}\right) \frac{1}{r^{2}} \frac{\partial^{2}}{\partial \theta^{2}} \\
& \left.\left(D_{66}-2 F_{66} c_{1}+H_{66} c_{1}^{2}\right) \frac{1}{r^{2}}-\left(A_{44}-2 D_{44} c_{2}+F_{44} c_{2}^{2}\right)\right) \phi_{\theta} \\
&
\end{aligned}
$$

The displacements and rotational functions are assumed in the separable form for annular plates as

$$
\begin{aligned}
u(r, \theta, t) & =U(r) \cos n \theta e^{i \omega t}, \\
v(r, \theta, t) & =V(r) \sin n \theta e^{i \omega t}, \\
w(r, \theta, t) & =W(r) \cos n \theta e^{i \omega t}, \\
\phi_{r}(r, \theta, t) & =\Phi_{r}(r) \cos n \theta e^{i \omega t}, \\
\phi_{\theta}(r, \theta, t) & =\Phi_{\theta}(r) \sin n \theta e^{i \omega t},
\end{aligned}
$$

where $r$ and $\theta$ are polar coordinates that describe the radial and circumferential direction, $\omega$ is the angular frequency of vibration, $t$ is the time, and $n$ is the circumferential node number.

The nondimensional parameters introduced are as follows:

$$
\begin{gathered}
R=\frac{r-a}{l}, \quad a \leq r \leq b, R \in[0,1], \\
\lambda=\omega l \sqrt{\frac{I_{1}}{A_{11}}}, \text { a frequency parameter, } \\
\beta=\frac{a}{b}, \text { the radii ratio, }
\end{gathered}
$$

$\gamma=\frac{h}{r_{a}}$, ratios of thickness to radius of inner circle,

$\delta_{k}=\frac{h_{k}}{h}$, relative layer thickness of the $k$-th layer.

Here, $h_{k}$ is the thickness of the $k$-th layer, $h$ is the total thickness of the plate, $r_{a}$ is the radius of inner circular plate, and $A_{11}$ is a standard extensional rigidity coefficient.

Using Equation (6) and introducing (12) and nondimensional parameters, the differential equations containing derivatives of the third order in $U(R)$, the second order in $V(R)$, the fourth order in $W(R)$, the third order in $\Phi_{R}(R)$, and the second order in $\Phi_{\theta}(R)$ are obtained.

2.1. Method of Solution. The differential equations containing derivatives of the third order in $U(R)$, the second order in $V(R)$, the fourth order in $W(R)$, the third order in $\Phi_{R}(R)$, and the second order in $\Phi_{\theta}(R)$. These functions are approximated by using cubic and quantic spline functions, in the range of $R \varepsilon[0,1]$, since splines are relatively simple and elegant and use a series of lower order approximations rather than global higher order approximations, affording fast convergence, and high accuracy.

The displacement functions $U(R), V(R)$, and $W(R)$ and the rotational functions $\Phi_{R}(R)$ and $\Phi_{\theta}(R)$ are approximated, respectively, by the splines. 
TABLE 1: Comparison of natural frequency parameter ( $\mathrm{rad} / \mathrm{s})$ of the present study $\lambda=\omega \ell \sqrt{I_{0} / A_{11}}$ with Hashemi et al. [53], Duan et al. [54], and Liu et al. [55] of simply supported annular circular plates $\beta=1 / 6, \gamma=1 / 60$.

\begin{tabular}{lccccc}
\hline $\begin{array}{l}\text { Circumferential node number } \\
n\end{array}$ & Modes & Present & Hashemi et al. [53] exact sol. & Duan et al. [54] FSDT & Liu et al. [55] FEM \\
\hline 1 & 1 & 1583 & 1584.65 & 1583 & 5433 \\
\\
2 & 5436 & 5437.61 & 11478 & 1551 \\
2 & 3 & 11494 & 11495.1 & 2306 & 6328 \\
& 1 & 2307 & 2308.11 & 6468 & 11283 \\
& 2 & 6474 & 6475.13 & 12632 & 12428 \\
\hline
\end{tabular}

TABLE 2: Variation of fundamental angular frequency $\omega$ with respect to radius ratio $\beta$ of 6-layered, 5-layered, and 3-layered annular circular plates for $n=4, n=2$, and $\gamma=0.05$ under simply supported boundary conditions.

\begin{tabular}{|c|c|c|c|c|c|c|}
\hline \multirow{3}{*}{$\beta$} & \multicolumn{3}{|c|}{$n=4$} & \multicolumn{3}{|c|}{$n=2$} \\
\hline & $0^{0} / 90^{0} / 0^{0} / 90^{\circ} / 0^{0} / 90^{0}$ & $0^{0} / 90^{0} / 0^{0} / 90^{\circ} / 0^{0}$ & $0^{0} / 90^{0} / 0^{0}$ & $0^{0} / 90^{0} / 0^{0} / 90^{0} / 0^{0} / 90^{0}$ & $0^{0} / 90^{0} / 0^{0} / 90^{\circ} / 0^{0}$ & $0^{0} / 90^{0} / 0^{0}$ \\
\hline & $G E / K E / G E / K E / G E / K E$ & $G E / K E / G E / K E / G E$ & $G E / K E / G E$ & $G E / K E / G E / K E / G E / K E$ & $G E / K E / G E / K E / G E$ & $G E / K E / G E$ \\
\hline 0.1 & 0.072388 & 0.061216 & 0.162124 & 0.456672 & 0.32758 & 0.331146 \\
\hline 0.3 & 3.20848 & 1.3214 & 1.5214 & 2.79001 & 4.71633 & 4.91633 \\
\hline 0.5 & 4.50102 & 3.21836 & 3.09182 & 5.12027 & 5.97262 & 7.2134 \\
\hline 0.7 & 11.6893 & 11.1519 & 12.79113 & 14.3642 & 10.498 & 13.4466 \\
\hline 0.9 & 44.5115 & 55.1115 & 103.567 & 49.1302 & 88.471 & 65.7481 \\
\hline
\end{tabular}

$$
\begin{gathered}
U(R)=\sum_{i=0}^{4} a_{i} R^{i}+\sum_{j=0}^{N-1} b_{j}\left(R-R_{j}\right)^{5} H\left(R-R_{j}\right), \\
V(R)=\sum_{i=0}^{2} c_{i} R^{i}+\sum_{j=0}^{N-1} d_{j}\left(R-R_{j}\right)^{3} H\left(R-R_{j}\right), \\
W(R)=\sum_{i=0}^{4} e_{i} R^{i}+\sum_{j=0}^{N-1} f_{j}\left(R-R_{j}\right)^{5} H\left(R-R_{j}\right), \\
\Phi_{X}(R)=\sum_{i=0}^{4} g_{i} R^{i}+\sum_{j=0}^{N-1} g_{j}\left(R-R_{j}\right)^{5} H\left(R-R_{j}\right), \\
\Phi_{Y}(R)=\sum_{i=0}^{2} l_{i} R^{i}+\sum_{j=0}^{N-1} q_{j}\left(R-R_{j}\right)^{3} H\left(R-R_{j}\right) .
\end{gathered}
$$

The resulting expressions contain $(5 N+5)$ homogeneous system of equations in the $(5 N+21)$ spline coefficients.

The boundary condition considered on the edges $R=0$ and $R=1$ are (S-S): both the ends simply supported

$$
V=W=\phi_{\theta}=N_{x}=M_{x}=N_{x \theta}=P_{x}=\bar{M}_{x}=0 \text { at } R=0, R=1 .
$$

This boundary condition gives 3 more equations, thus making a total of $(5 N+18)$ equations, in the same number

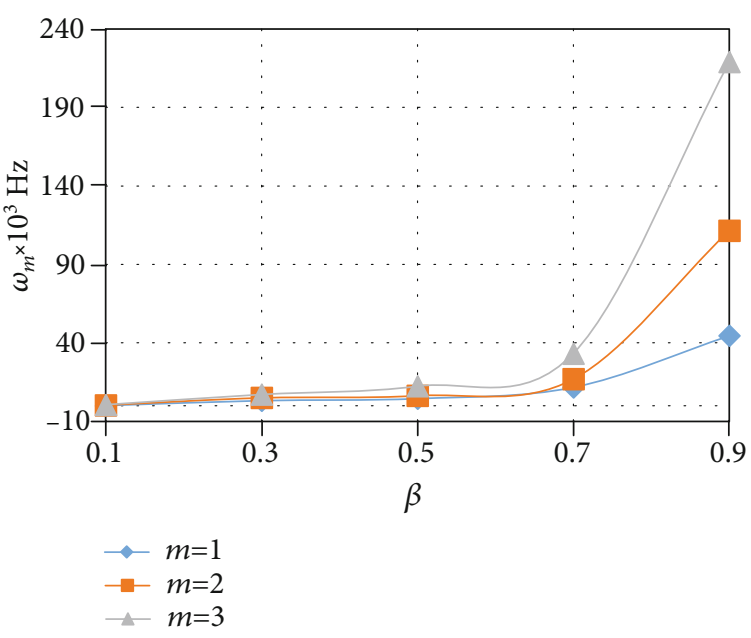

FIgURE 3: Variation of angular frequency and radius ratio of 6layered annular circular plates. S-S.

of unknowns. The resulting field and boundary condition equations may be written in the form

$$
[M]\{q\}=\lambda^{2}[P]\{q\}
$$

where $[M]$ and $[P]$ are square matrices and $\{q\}$ is a column matrix. This is treated as a generalized eigenvalue problem 


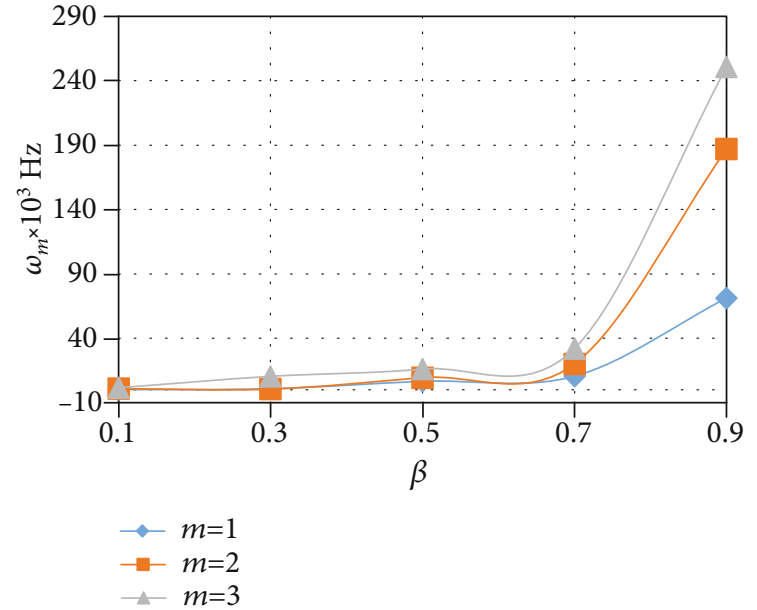

FIgURE 4: Variation of angular frequency and radius ratio of 4layered annular circular plates. S-S.

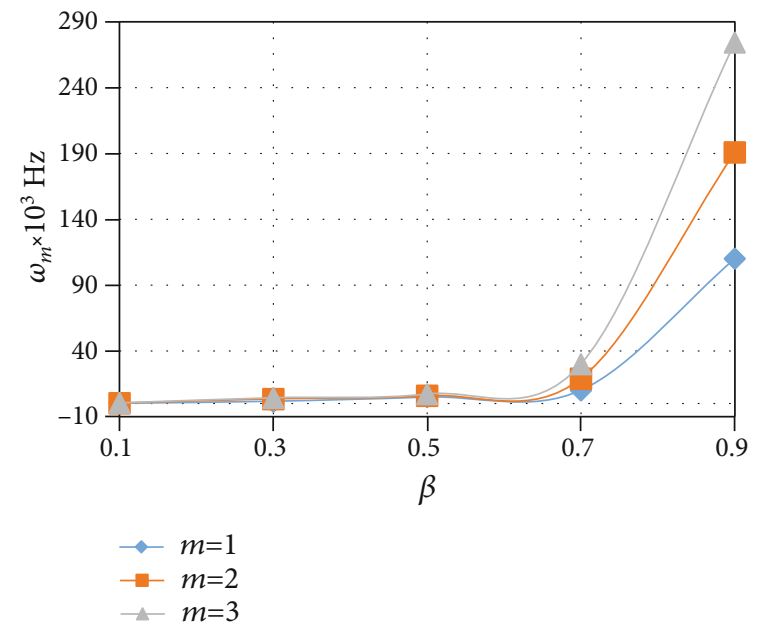

FIGURE 5: Variation of angular frequency and radius ratio of 2layered annular circular plates. S-S.

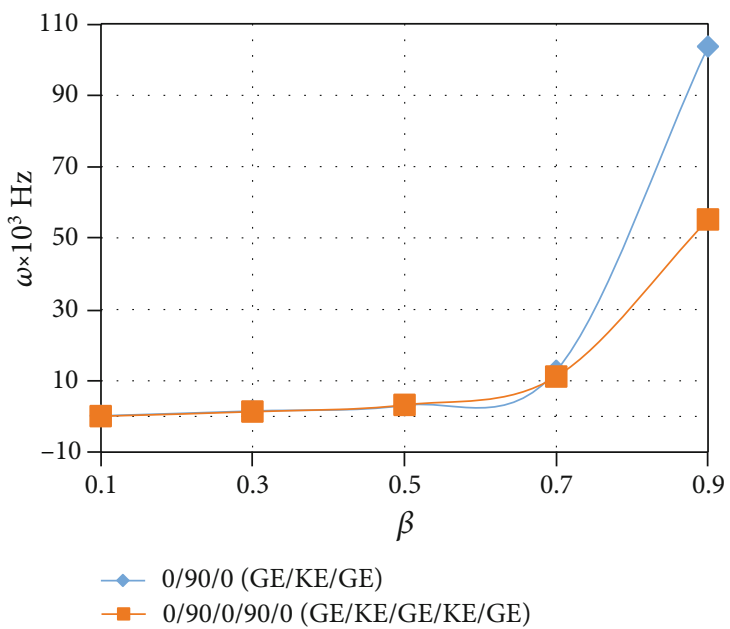

Figure 6: Variation of angular frequency and radius ratio of 3- and 5-layered annular circular plates. S-S.

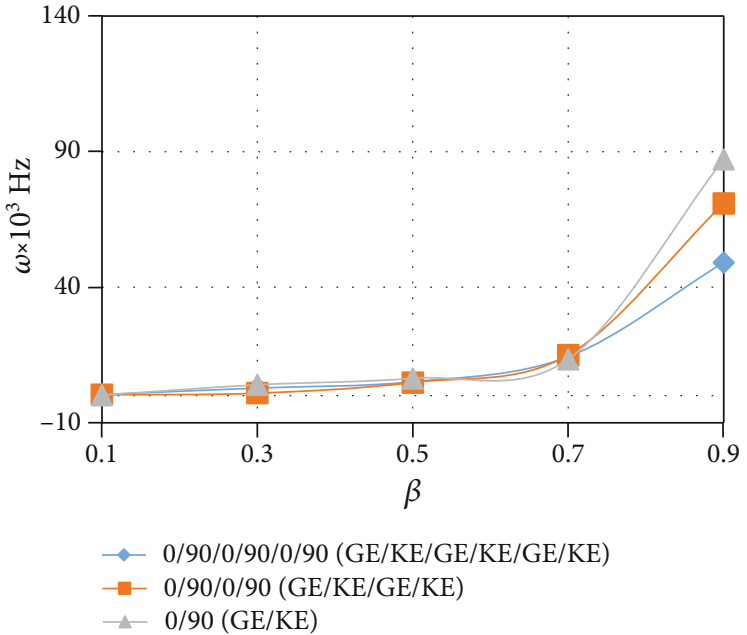

FIGURE 7: Variation of angular frequency and radius ratio of 6-, 4-, and 2-layered annular circular plates. S-S.

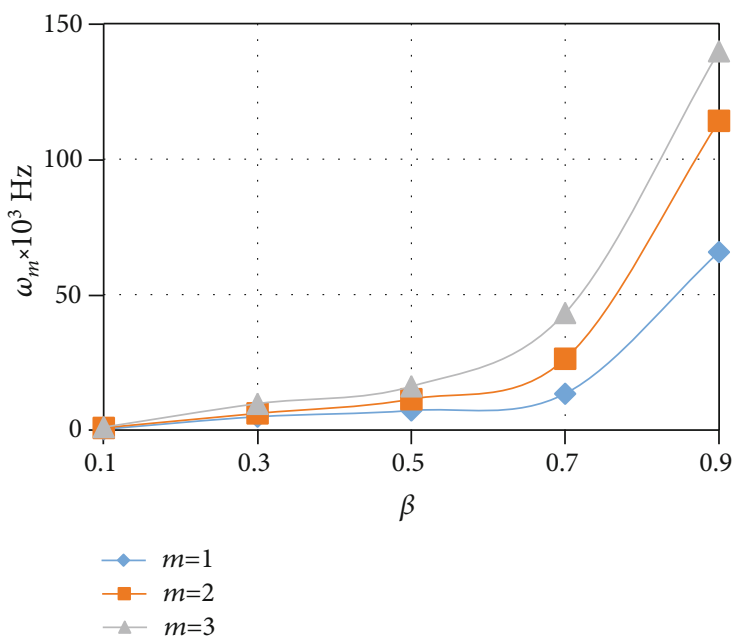

FIGURE 8: Variation of angular frequency and radius ratio of 3layered annular circular plates. S-S.

in the eigenparameter $\lambda$ and the eigenvector $\{q\}$ whose elements are the spline coefficients.

\section{Results and Discussion}

The annular circular plates for simply supported boundary condition based on the higher order shear deformation theory are investigated. All numerical computations, unless otherwise stated, two materials are considered: kevlar-49/ epoxy (KE) and graphite/epoxy (AS4/3501-6) (GE). Annular circular plates of two, three, four, five, and six layers with cross-ply orientations are considered for analysis.

3.1. Convergence Study. In this subsection, the frequency parameter with respect to different configurations is carried out to confirm the convergence of spline method for conical shells. The number of subintervals $N$ of the range $R \in[0,1]$. 


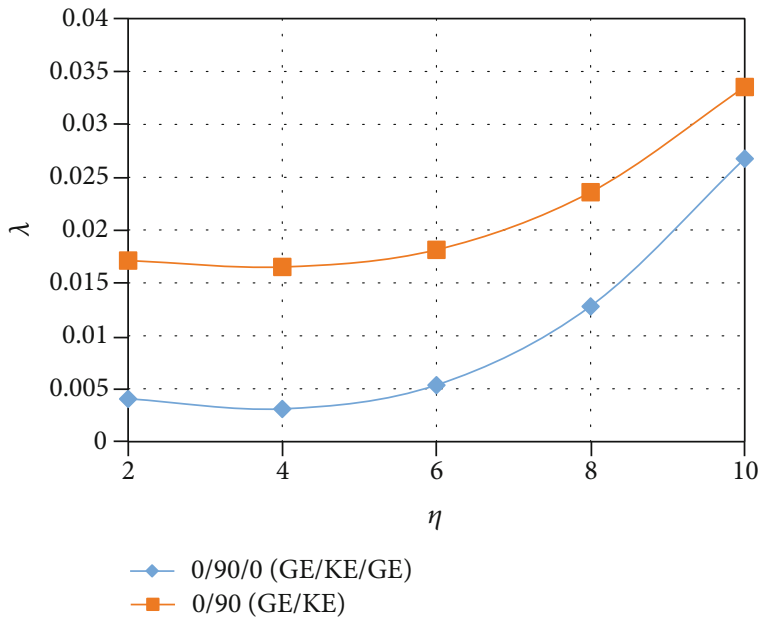

Figure 9: Variation of fundamental frequency parameter and circumferential node number of 3- and 2-layered annular circular plates. S-S.

The value of $N$ started from 2 , and finally, it is fixed for $N$ $=14$, since for the next value of $N$, the percent changes in the values of $\lambda$ are very low, the maximum being $3 \%$.

3.2. Validation. Table 1 shows the comparison of the reduced case of the current results with Hashemi et al. [53], Duan et al. [54], and Liu et al. [55] for simply supported annular circular plates, which is the validation of the applicability and accuracy of present results with the available results. The pfresent results are close to the available results.

3.3. Result and Discussion. Table 2 illustrates the variation of angular frequency $\omega$ and radius ratio $\beta$ of $6-, 5-$, and 3layered annular circular plates for $\gamma=0.05$ and $n=4$ and $n$ $=2$, concluding that angular frequency increases slowly between $\beta=0.1$ and $\beta=0.5$ and increases significantly between $\beta=0.5$ and $\beta=0.9$.

Figure 3 depicts the variation of angular frequency and radius ratio of 6-layered annular circular plates for circumferential node number and ratio of thickness to radius of inner circle as $n=4, \gamma=0.05$. It can be seen that angular frequency remains almost same until $\beta=0.7$ and increases significantly afterwards for all modes. Figure 4 shows the variation of angular frequency and radius ratio of 4-layered annular circular plates. The angular frequency remains almost the same for a certain value of $\beta$ and increases significantly afterwards. The variation of angular frequency and radius ratio of 2-layered annular circular plates are shown in Figure 5. The circumferential node number and ratio of thickness to radius of inner circle considered are $n=4, \gamma=0.05$. The frequency remains almost the same until $\beta=0.7$ and increases afterwards.

Figure 6 shows the variation of angular frequency and radius ratio of 3 - and 5-layered annular circular plates with $n=4, \gamma=0.05$, showing that angular frequency for three-layered annular circular plates is higher than that for five-layered annular circular plates between $\beta=0.7$

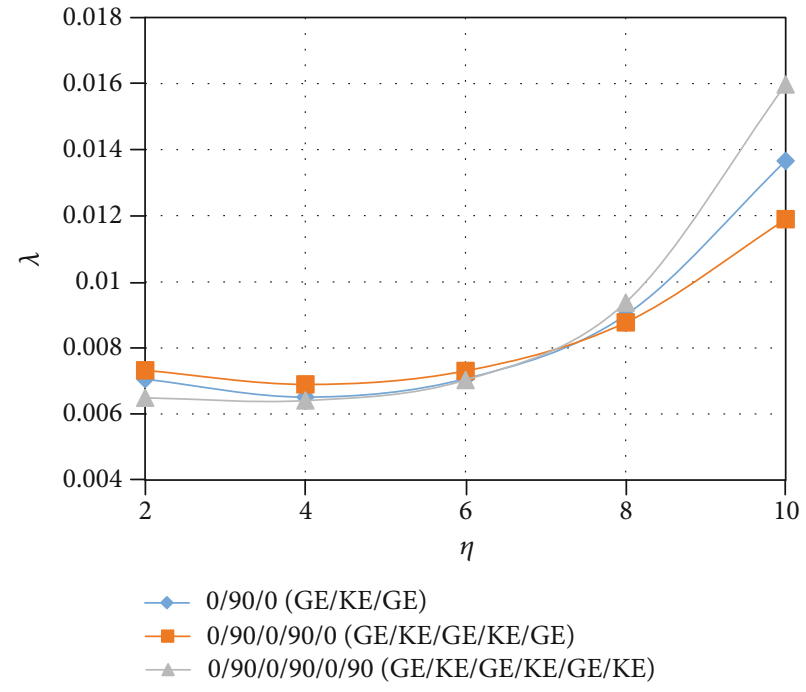

FIGURE 10: Variation of angular frequency and circumferential node number of 3-, 5-, and 6-layered annular circular plates. S-S.

and $\beta=0.9$.Variation of angular frequency and radius ratio can be seen in Figure 7 for 6-, 4-, and 2-layered annular circular plates with $n=2, \gamma=0.05$. The figure shows that angular frequency for two-layered annular circular plates is the highest followed by four- and sixlayered annular circular plates between $\beta=0.7$ and $\beta=$ 0.9 , concluding that the angular frequency value increases as the number of layers decreases.

Figure 8 shows the variation of angular frequency and radius ratio of 3 -layered $\left(0^{0} / 90^{\circ} / 0^{\circ}(G E / K E / G E)\right)$ annular circular plates with $n=2, \gamma=0.05$, showing that angular frequency for three modes is almost the same between $\beta$ $=0.1$ and $\beta=0.5$ and the difference of angular frequency for three modes increases and becomes higher between $\beta=0.6$ and $\beta=0.9$.

Figure 9 shows the variation of fundamental frequency parameter and circumferential node number of 3- and 2layered $\left(0^{\circ} / 90^{\circ} / 0^{\circ}(G E / K E / G E)\right.$ and $\left.0^{\circ} / 90^{\circ}(G E / K E)\right)$ annular circular plates with $\beta=0.3, \gamma=0.05$ showing that the frequency value gradually increases with the increase of circumferential node number with 3-layered annular circular plate frequency which is lower than 2-layered annular circular plate frequency.

Figure 10 shows the variation of fundamental frequency parameter and circumferential node number of 3-, 5-, and 6layered $\left(0^{0} / 90^{\circ} / 0^{0}(\mathrm{GE} / \mathrm{KE} / \mathrm{GE}), 0^{0} / 90^{\circ} / 0^{\circ} / 90^{\circ} / 0^{\circ}(\mathrm{GE} / \mathrm{KE} / \mathrm{G}\right.$ $E / K E / G E)$, and $0^{0} / 90^{\circ} / 0^{\circ} / 90^{\circ} / 0^{\circ} / 90^{\circ}(G E / K E / G E / K E / G E / K$ $E)$ ) annular circular plates with $\beta=0.5, \gamma=0.05$ showing that frequency value gradually increases with the increase of circumferential node number.

The first three mode shapes of vibration of cross-ply 2-layered $\left(0^{\circ} / 90^{\circ}(\mathrm{GE} / \mathrm{KE})\right)$ annular circular plates is presented in Figures 11-13(a)-13(c). Normalization is done with respect to the maximum transverse displacement $W$. As expected, the transverse displacements are the most predominant. 


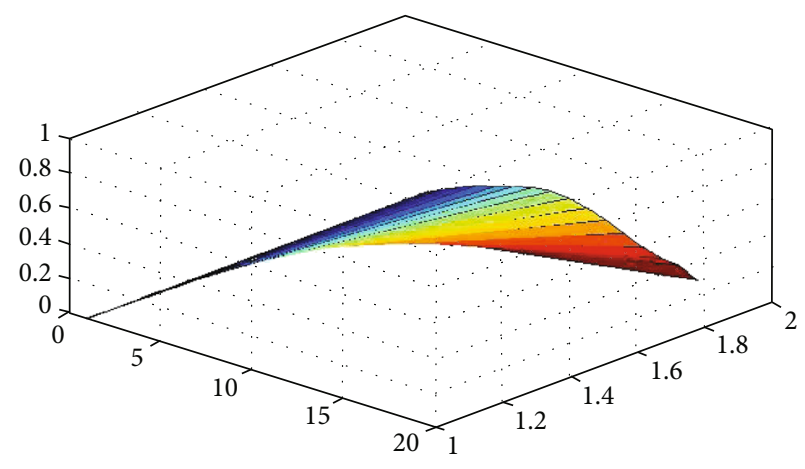

(a)

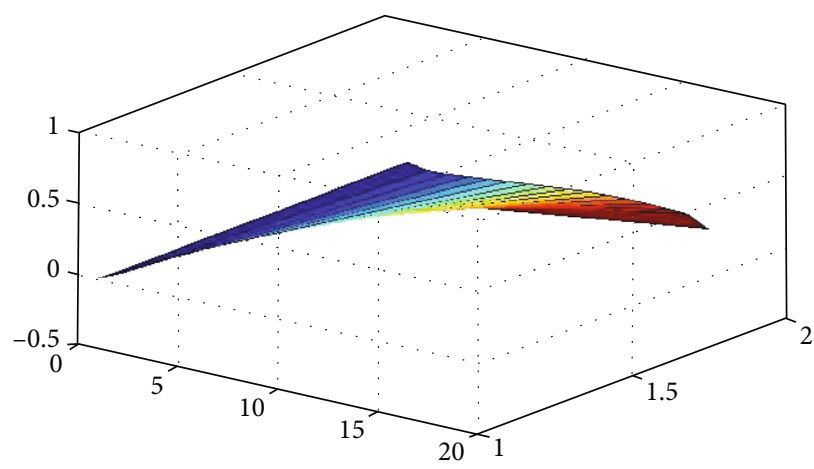

(b)

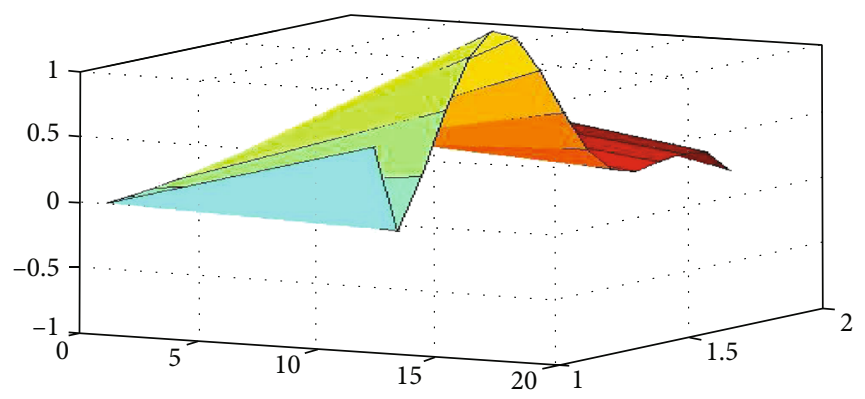

(c)

FIGURE 11: (a-c) Mode shapes of cross-ply annular circular plate $(U)$.

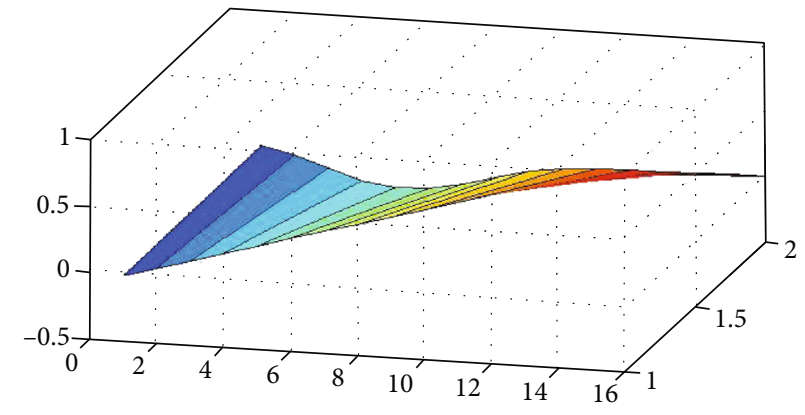

(a)

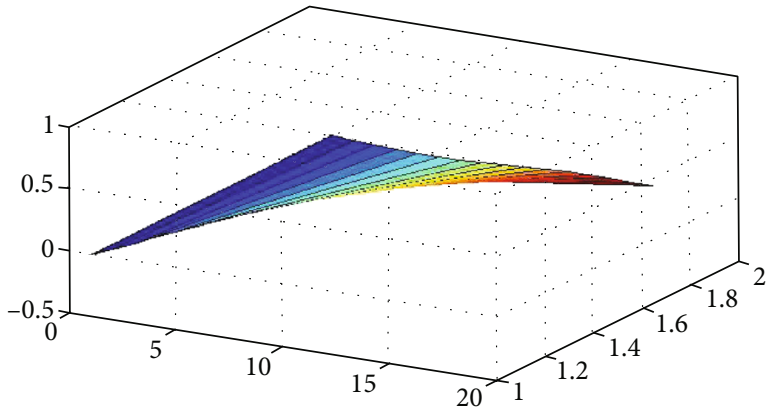

(b)

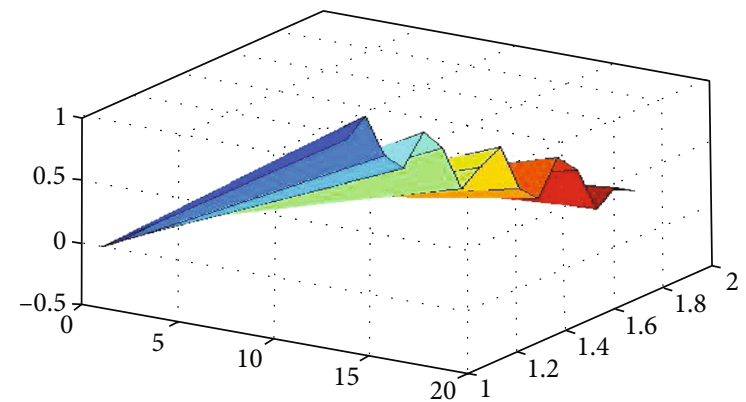

(c)

FIgURE 12: (a-c) Mode shapes of cross-ply annular circular plate $(V)$. 


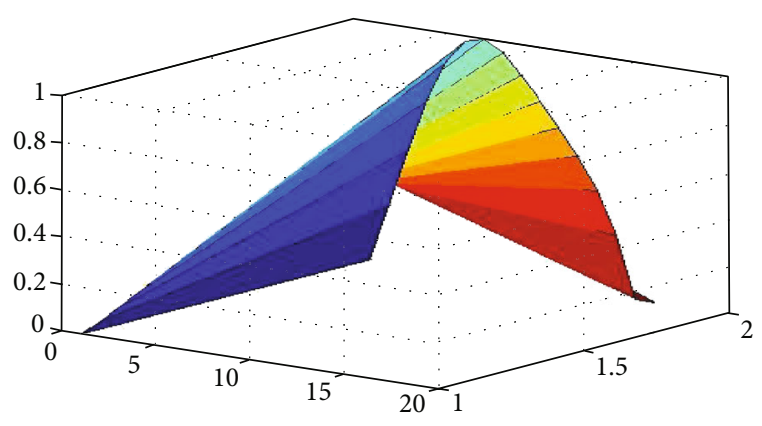

(a)

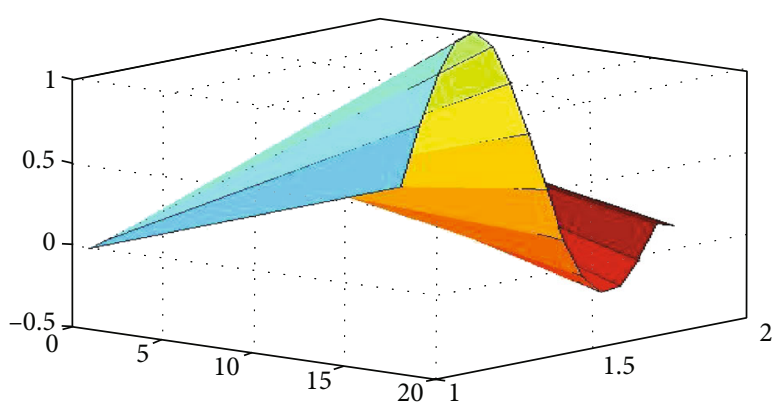

(b)

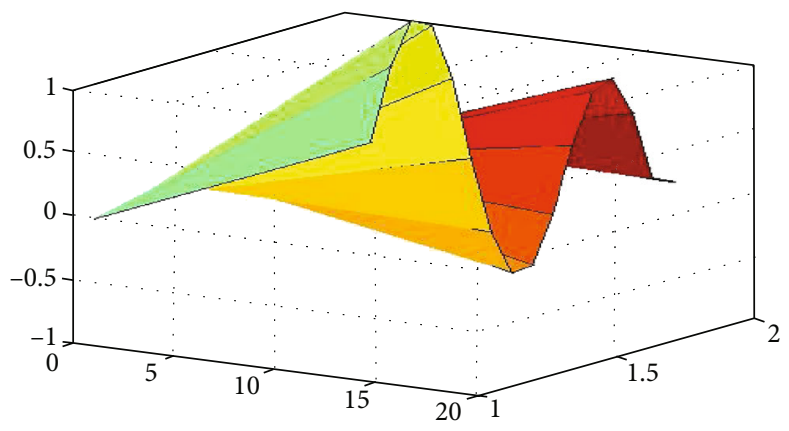

(c)

FIGURE 13: (a-c) Mode shapes of cross-ply annular circular plate $(W)$.

\section{Conclusion}

The free vibration analysis of annular circular plates based on higher-order shear deformation theory using spline approximation under simply supported boundary condition is investigated. The frequency variation of annular circular plates is studied for circumferential node number, radius ratio, different lamination schemes, and materials used. The effect of radius ratio on the angular frequency is that angular frequency remains almost the same until $\beta=0.7$ and increases significantly afterwards for all modes. The effect of circumferential node number on the fundamental frequency parameter is that the frequency value gradually increases with the increase of circumferential node number. Results obtained by the present technique will be helpful for future researchers to validate their results.

\section{Appendix}

Stiffness coefficients are

$$
\begin{aligned}
A_{i j} & =\sum_{k} \bar{Q}_{i j}^{(k)}\left(z_{k}-z_{k-1}\right), B_{i j} \\
& =\frac{1}{2} \sum_{k} \bar{Q}_{i j}^{(k)}\left(z_{k}^{2}-z_{k-1}^{2}\right), D_{i j} \\
& =\frac{1}{3} \sum_{k} \bar{Q}_{i j}^{(k)}\left(z_{k}^{3}-z_{k-1}^{3}\right),
\end{aligned}
$$

$$
\begin{aligned}
E_{i j} & =\frac{1}{4} \sum_{k} \bar{Q}_{i j}^{(k)}\left(z_{k}^{4}-z_{k-1}^{4}\right), F_{i j} \\
& =\frac{1}{5} \sum_{k} \bar{Q}_{i j}^{(k)}\left(z_{k}^{5}-z_{k-1}^{5}\right), H_{i j} \\
& =\frac{1}{7} \sum_{k} \bar{Q}_{i j}^{(k)}\left(z_{k}^{7}-z_{k-1}^{7}\right) .
\end{aligned}
$$

For $i, j=1,2,6$,

$$
\begin{aligned}
A_{i j}{ }^{\prime} & =\sum_{k} \bar{Q}_{i j}^{(k)}\left(z_{k}-z_{k-1}\right), D_{i j}{ }^{\prime} \\
& =\frac{1}{3} \sum_{k} \bar{Q}_{i j}^{(k)}\left(z_{k}^{3}-z_{k-1}^{3}\right), F_{i j}{ }^{\prime} \\
& =\frac{1}{5} \sum_{k} \bar{Q}_{i j}^{(k)}\left(z_{k}^{5}-z_{k-1}^{5}\right),
\end{aligned}
$$

for $i, j=4,5$.

\section{Data Availability}

The data supporting the results of this study are included in the manuscript.

\section{Conflicts of Interest}

The authors declare that they have no conflict of interest regarding the publication of this paper. 


\section{Acknowledgments}

The authors gratefully acknowledge the Deanship of Scientific Research, King Faisal University, Saudi Arabia, for the financial support under Nasher Track (Grant No. NA00034).

\section{References}

[1] J. R. Vinson, "Sandwich Structures," Applied Mechanics Reviews, vol. 54, no. 3, pp. 201-214, 2001.

[2] A. K. Noor, W. S. Burton, and C. W. Bert, "Computational models for sandwich panels and shells," Applied Mechanics Reviews, vol. 49, no. 3, pp. 155-199, 1996.

[3] P. C. Yang, C. H. Nooris, and Y. Stavsky, "Elastic wave propagation in heterogeneous plates," International Journal of Solids and Structures, vol. 2, no. 4, pp. 665-684, 1966.

[4] P. F. Pai, "A new look at shear correction factors and warping functions of anisotropic laminates," International Journal of Solids and Structures, vol. 32, no. 16, pp. 2295-2313, 1995.

[5] P. F. Pai and M. J. Schulz, "Shear correction factors and an energy-consistent beam theory," International Journal of Solids and Structures, vol. 36, no. 10, pp. 1523-1540, 1999.

[6] J. N. Reddy, Theory and Analysis of Elastic Plates and Shells, CRC press, 2006.

[7] A. W. Leissa, Vibrations of Plates, NASA SP-160, USA, 1969.

[8] A. W. Leissa, "Recent research in plate vibrations: classical theory," The Shock and Vibration Digest, vol. 9, pp. 13-24, 1977.

[9] A. W. Leissa, "Plate vibration research, 1976-1980: classical theory," The Shock and Vibration Digest, vol. 13, pp. 11-22, 1981.

[10] A. W. Leissa, "Recent studies in plate vibrations: 1981-1985, part I: classical theory," The Shock and Vibration Digest, vol. 19, pp. 11-18, 1987.

[11] C. Dongxing and G. Yanhui, "Free vibration of non-uniform axially functionally graded beams using the asymptotic development method," Applied Mathematics and Mechanics, vol. 40, no. 1, pp. 85-96, 2019.

[12] S. Azaripour and M. Baghani, "Vibration analysis of FG annular sector in moderately thick plates with two piezoelectric layers," Applied Mathematics and Mechanics, vol. 40, no. 6, pp. 783-804, 2019.

[13] Y. Xue, G. Jin, H. Ding, and M. Chen, "Free vibration analysis of in-plane functionally graded plates using a refined plate theory and isogeometric approach," Composite Structures, vol. 192, pp. 193-205, 2018.

[14] R. Zhong, Q. Wang, J. Tang, C. Shuai, and B. Qin, "Vibration analysis of functionally graded carbon nanotube reinforced composites (FG-CNTRC) circular, annular and sector plates," Composite Structures, vol. 194, pp. 49-67, 2018.

[15] E. Arshid and A. R. Khorshidvand, "Free vibration analysis of saturated porous FG circular plates integrated with piezoelectric actuators via differential quadrature method," ThinWalled Structures, vol. 125, pp. 220-233, 2018.

[16] K. K. Żur, "Free vibration analysis of elastically supported functionally graded annular plates via quasi-Green's function method," Composites Part B: Engineering, vol. 144, pp. 37$55,2018$.

[17] C. S. Kim and S. M. Dickinson, "On the lateral vibration of thin annular and circular composite plates subject to certain com- plicating effects," Journal of Sound and Vibration, vol. 130, no. 3, pp. 363-377, 1989.

[18] C. S. Kim and S. M. Dickinson, "The flexural vibration of thin isotropic and polar orthotropic annular and circular plates with elastically restrained peripheries," Journal of Sound and Vibration, vol. 143, no. 1, pp. 171-179, 1990.

[19] X. Wang, A. G. Striz, and C. W. Bert, "Free vibration analysis of annular plates by the DQ method," Journal of Sound and Vibration, vol. 164, no. 1, pp. 173-175, 1993.

[20] C. F. Liu and G. T. Chen, "A simple finite element analysis of axisymmetric vibration of annular and circular plates," International Journal of Mechanical Sciences, vol. 37, no. 8, pp. 861-871, 1995.

[21] U. S. Gupta, R. Lal, and S. Sharma, "Vibration of nonhomogeneous circular Mindlin plates with variable thickness," Journal of Sound and Vibration, vol. 302, no. 1-2, pp. 1-17, 2007.

[22] E. Romanelli, R. E. Rossi, P. A. A. Laura, and R. H. Gutierrez, "Transverse vibration sofa circular annular plate with an intermediate circular support and a free inner edge," Journal of Sound and Vibration, vol. 212, no. 3, pp. 564-571, 1996.

[23] P. A. A. Laura, R. H. Gutierrez, and R. E. Rossi, "Transverse vibrations of a circular, annular plate with free edges and two, intermediate concentric circular supports," Journal of Sound and Vibration, vol. 226, no. 5, pp. 1043-1047, 1999.

[24] S. A. Vera, L. PAA, and D. A. Vega, "Transverse vibrations of a free-free circular annular plate," Journal of Sound and Vibration, vol. 224, no. 2, pp. 379-383, 1999.

[25] E. Efraim and M. Eisenberger, "Exact vibration analysis of variable thickness thick annular isotropic and FGM plates," Journal of Sound and Vibration, vol. 299, no. 4-5, pp. 720738, 2007.

[26] C. Y. Wang, "Vibration of an annular membrane attached to a free, rigid core," Journal of Sound and Vibration, vol. 260, no. 4, pp. 776-782, 2003.

[27] Z. Celep and D. Turhan, "Axisymmetric vibrations of circular plates on tensionless elastic foundations," Journal of Applied Mechanics, vol. 57, no. 3, pp. 677-681, 1990.

[28] M. Utku, E. Citipitioğlu, and I. Inceleme, "Circular plates on elastic foundations modelled with annular plates," Computers \& Structures, vol. 78, no. 1-3, pp. 365-374, 2000.

[29] S. Kukla and M. Szewczyk, "Free vibration of annular plates of stepped thickness resting on Winkler elastic foundation," Scientific Research of the Institute of Mathematics and Computer Science, vol. 6, no. 1, pp. 109-116, 2007.

[30] R. P. Singh and S. K. Jain, "Free asymmetric transverse vibration of parabolically varying thickness polar orthotropic annular plate with flexible edge conditions," Journal of Applied Science and Engineering, vol. 7, pp. 41-52, 2004.

[31] R. P. Singh and S. K. Jain, "Free asymmetric transverse vibration of polar orthotropic annular sector plate with thickness varying parabolically in radial direction," Sadhana, vol. 29, no. 5, pp. 415-428, 2004.

[32] V. Tajeddini, A. Ohadi, and A. Sadighi, "Three-dimensional free vibration of variable thickness thick circular and annular isotropic and functionally graded plates on Pasternak foundation," International Journal of Mechanical Sciences, vol. 53, no. 4, pp. 300-308, 2011.

[33] H. Ersoy, K. Mercan, and Ö. Civalek, "Frequencies of FGM shells and annular plates by the methods of discrete singular 
convolution and differential quadrature methods," Composite Structures, vol. 183, pp. 7-20, 2018.

[34] F. Tornabene, "Free vibration analysis of functionally graded conical, cylindrical shell and annular plate structures with a four-parameter power-law distribution," Computer Methods in Applied Mechanics and Engineering, vol. 198, no. 37-40, pp. 2911-2935, 2009.

[35] F. Tornabene, E. Viola, and D. J. Inman, "2-D differential quadrature solution for vibration analysis of functionally graded conical, cylindrical shell and annular plate structures," Journal of Sound and Vibration, vol. 328, no. 3, pp. 259-290, 2009.

[36] T. V. Vu, A. Khosravifard, M. R. Hematiyan, and T. Quocbui, "A new refined simple TSDT-based effective meshfree method for analysis of through-thickness FG plates," Applied Mathematical Modelling, vol. 57, pp. 514-534, 2018.

[37] C. H. Thai, L. V. Tran, D. T. Tran, T. Nguyen-Thoi, and H. Nguyen-Xuan, "Analysis of laminated composite plates using higher-order shear deformation plate theory and node-based smoothed discrete shear gap method," Applied Mathematical Modelling, vol. 36, no. 11, pp. 5657-5677, 2012.

[38] S. Hosseini-Hashemi, H. Rokni Damavandi Taher, M. Akhavan, and M. Omidi, "Free vibration of functionally graded rectangular plates using first-order shear deformation plate theory," Applied Mathematical Modelling, vol. 34, no. 5, pp. 1276-1291, 2010.

[39] S. Hosseini-Hashemi, M. Es'Haghi, H. R. Taher, and M. Fadaie, "Exact closed-form frequency equations for thick circular plates using a third-order shear deformation theory," Journal of Sound and Vibration, vol. 329, no. 16, pp. i3382i3396, 2010.

[40] T. K. Nguyen, H. T. Thai, and T. P. Vo, "A novel general higher-order shear deformation theory for static, vibration and thermal buckling analysis of the functionally graded plates," Journal of Thermal Stresses, vol. 44, no. 3, pp. 377394, 2020.

[41] A. A. Daikh, A. Drai, I. Bensaid, M. S. Houari, and A. Tounsi, "On vibration of functionally graded sandwich nanoplates," Journal of Sandwich Structures \& Materials, vol. 23, no. 6, pp. 2217-2244, 2020.

[42] A. Baghlani, M. Khayat, and S. M. Dehghan, "Free vibration analysis of fgm cylindrical shells surrounded by pasternak elastic foundation in thermal environment considering fluidstructure interaction," Applied Mathematical Modelling, vol. 78, pp. 550-575, 2020.

[43] I. Katili, J. L. Batoz, I. J. Maknun, and A. M. Katili, “On static and free vibration analysis of FGM plates using an efficient quadrilateral finite element based on DSPM," Composite Structures, vol. 261, article 113514, 2021.

[44] R. S. Dhari, "A numerical study on cross ply laminates subjected to stray fragments impact loading," Composite Structures, vol. 261, article 113563, 2021.

[45] M. Li, R. Yan, L. Xu, and C. Guedes Soares, “A general framework of higher-order shear deformation theories with a novel unified plate model for composite laminated and FGM plates," Composite Structures, vol. 261, article 113560, 2021.

[46] E. Askari, K. H. Jeong, and M. Amabili, “A novel mathematical method to analyze the free vibration of eccentric annular plates," Journal of Sound and Vibration, vol. 484, article 115513, 2020.
[47] M. Amabili, G. Frosali, and M. K. Kwak, "Free vibrations of annular plates coupled with fluids," Journal of Sound and Vibration, vol. 191, no. 5, pp. 825-846, 1996.

[48] S. Javed, K. K. Viswanathan, Z. A. Aziz, and K. Prabakar, "Free vibration of anti-symmetric angle-ply plates with variable thickness," Composite iStructures, vol. 137, pp. 56-69, 2016.

[49] S. Javed, K. K. Viswanathan, Z. A. Aziz, K. Karthik, and J. H. Lee, "Vibration of antisymmetric angle-ply laminated plates under higher order shear theory," Steel and composite structures, vol. 22, no. 6, pp. 1281-1299, 2016.

[50] S. Javed, K. K. Viswanathan, M. N. Izyan, Z. A. Aziz, and J. H. Lee, "Free vibration of cross-ply laminated plates based on higher-order shear deformation theory," Steel and composite structures, vol. 26, no. 4, pp. 473-484, 2018.

[51] S. Javed, "Free vibration characteristic of laminated conical shells based on higher- order shear deformation theory," Composite Structures, vol. 204, pp. 80-87, 2018.

[52] M. Toorani and A. A. Lakis, "General equations of anisotropic plates and shells including transverse shear deformations, rotary inertia and initial curvature effects," Journal of Sound and Vibration, vol. 237, no. 4, pp. 561-615, 2000.

[53] H. S. Hashemi, M. Es'haghi, and H. R. D. Taher, “An exact analytical solution for freely vibrating piezoelectric coupled circular/annular thick plates using Reddy plate theory," Composite Structures, vol. 92, pp. 1333-1351, 2010.

[54] W. H. Duan, S. T. Quek, and Q. Wang, "Free vibration analysis of piezoelectric coupled thin and thick annular plate," Journal of Sound and Vibration, vol. 281, pp. 119-139, 2005.

[55] C. F. Liu, T. J. Chen, and Y. J. Chen, "A modified axisymmetric finite element for the 3-D vibration analysis of piezoelectric laminated circular and annular plates," Journal of Sound and Vibration, vol. 309, pp. 794-804, 2008. 